\title{
Results of the first year of extragalactic observations with MAGIC
}

Javier Rico*

Institut de Física d'Altes Energies, Universitat Autònoma de Barcelona, 08193, Bellaterra, Spain

E-mail: jricodifae.es

\section{Roger Firpo}

Institut de Física d'Altes Energies, Universitat Autònoma de Barcelona, 08193, Bellaterra, Spain E-mail: rfirpodifae.es

\section{on behalf of the MAGIC Collaboration ${ }^{\dagger}$}

URL: http: //magic.mppmu.mpg.de/

The Major Atmospheric Gamma-ray Imaging Cherenkov (MAGIC) telescope is the largest, single-dish, imaging air cherenkov telescope (IACT), thus yielding the lowest energy threshold $(E \geq 50 \mathrm{GeV})$. The first cycle of observations has spanned the period between March 2005 and April 2006. About $75 \%$ of the observation time has been devoted to extragalactic objects. In this paper we briefly review the most important results of this first cycle on active galactic nuclei (AGN) and gamma ray bursts (GRB)

VI Microquasar Workshop: Microquasars and Beyond

September 18-22 2006

Società del Casino, Como, Italy

\footnotetext{
${ }^{*}$ Speaker.

†We thank the Instituto de Astrofísica de Canarias for the excellent working conditions at the Observatory Roque de los Muchachos in La Palma
} 


\section{Introduction}

MAGIC is a telescope for very high energy (VHE, $E \geq 50 \mathrm{GeV}$ ) gamma-ray observation exploiting the Imaging Air Cherenkov technique. It is located on the Roque de los Muchachos Observatory $\left(28^{\circ} 45^{\prime} 30^{\prime \prime} \mathrm{N}, 17^{\circ} 52^{\prime} 48^{\prime \prime} \mathrm{W}, 2250 \mathrm{~m}\right.$ above see level) in La Palma (Spain). This kind of instrument images the Cherenkov light produced in the particle cascade initiated by a gamma ray in the atmosphere. MAGIC incorporates a number of technological improvements in its design and is currently the largest single-dish telescope (diameter $17 \mathrm{~m}$ ) in this energy band, yielding the lowest threshold $(\sim 50 \mathrm{GeV})$. It is equipped with a 576-pixel photomultiplier camera with a $3.5^{\circ}$ fi eld of view. MAGIC's sensitivity above $100 \mathrm{GeV}$ is $\sim 2.5 \%$ of the Crab nebula flux (the calibration standard candle for IACTs) in 50 hours of observations. The relative energy resolution above $200 \mathrm{GeV}$ is better than $30 \%$. The angular resolution is $\sim 0.1^{\circ}$, while source localization in the sky is provided with a precision of $\sim 2^{\prime}$. MAGIC has the capability of being repositioned to any point in the sky within $40 \mathrm{~s}$ on average, which allows the observation of GRBs while still in the prompt emission. MAGIC is also unique among IACTs by its capability to operate under moderate illumination (i.e. moonlight and twilight). This allows to increase the duty cycle by a factor 1.5 and a better sampling of variable sources is possible.

\section{Observation of VHE gamma-ray candidate blazars}

All known VHE gamma-ray emitting blazars belong to the class of high-frequency-peaked BL Lacertae objects (HBLs, [1]), a subclass of blazars characterized by a low luminosity when compared with quasars and a synchrotron peak in the X-ray band. Their Spectral Energy Distribution (SED) is characterized by a second peak at very high gamma-ray energies. In synchrotron-selfCompton (SSC) models it is assumed that the observed gamma-ray peak is due to the inverseCompton (IC) emission from the accelerated electrons up-scattering previously produced synchrotron photons to high energies [2]. A compilation of blazars with known X-ray spectra allowing their classifi cation as HBLs is given in [3].

The MAGIC telescope observed a sample of X-ray bright $\left(F_{1 \mathrm{keV}}>2 \mu \mathrm{Jy}\right)$ northern HBLs at moderate redshifts $(z<0.3)$. The sample of candidates was chosen based on predictions from models involving an SSC [4] and hadronic [5] origin of the gamma rays. Also, since the known VHE gamma-ray emitting AGNs are variable in flux in all wavebands, the MAGIC collaboration has performed Target of Opportunity observations whenever they were alerted about sources being in a high flux state in the optical and/or X-ray band.

1ES 1218+30.4. This distant $(z=0.182)$ blazar is the fi rst source discovered by MAGIC, and the second furthest object detected at VHE. It had been previously observed by both Whipple and HEGRA collaborations, but only upper limits were derived. MAGIC observed it for $8.2 \mathrm{~h}$, obtaining a clear gamma-ray signal with a $6.4 \sigma$ signifi cance for energies above $140 \mathrm{GeV}$. The measured and intrinsic VHE differential spectrum is shown in Figure 1. No signifi cant day to day flux variations were observed during the observation period. For the details of the analysis and results see [6].

PG 1553+113. This source belongs to a catalog of X-ray bright objects [3] and, based on its SED properties, was one of the most promising candidates from a list of VHE gamma-ray emitting 


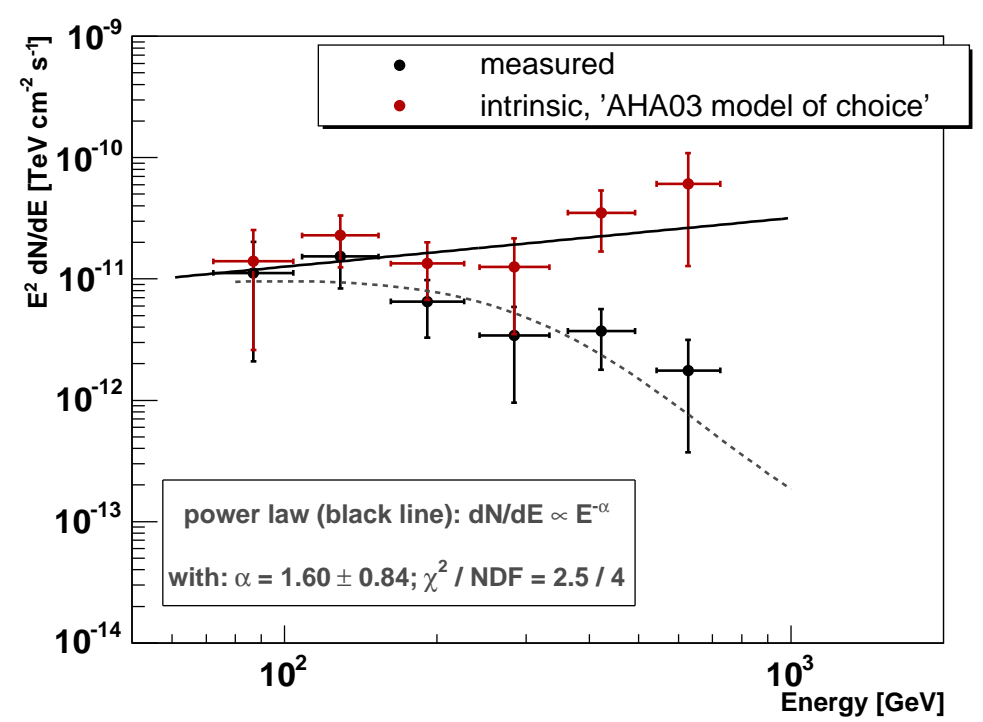

Figure 1: Measured (black points) and intrinsic (red points) differential energy spectrum of 1ES 1218+304. Black line: pure power law fit to the intrinsic spectrum, the fit parameters are listed in the inlay.

AGNs proposed by [4]. A hint of a detection ( $4 \sigma$ level) was recently reported by the H.E.S.S. collaboration [7]. PG 1553+113 was observed with the MAGIC telescope in 2005 and 2006 and a very clear gamma-ray signal was detected with a total signifi cance of $8.8 \sigma$. There is no evidence of a short term variability on a time scale of days, but a signifi cant change by a factor of three in the flux level from 2005 to 2006 was found. The combined 2005 and 2006 differential energy spectrum for PG $1553+113$ is well described by a pure power law with a photon index $\alpha=4.2 \pm 0.4$. Details of the analysis and results can be found at [8].

Mkn 180. This object is a well known high frequency peaked BL Lac (HBL) but a VHE gamma-ray emission had never been detected before. Its SED shows a generic two-bump distribution with maxima at X-ray and the GeV-TeV band. The Target of Opportunity observation of this source by MAGIC was triggered by a strong optical outburst detected by the KVA telescope. It was observed for a total of $11.1 \mathrm{~h}$, and gamma-ray emission was detected with $5.5 \sigma$ signifi cance. Details of the analysis can be found at [9].

1ES 2344+514. This gamma-ray source was fi rst detected in flaring state by Whipple, and later HEGRA reported a detection in the quiescent state. MAGIC has observed this source for $27.6 \mathrm{~h}$, and measured a gamma-ray signal with a signifi cance of $11.5 \sigma$. The source was in a quiescent state during the the observations, with a flux level compatible with HEGRA results, but a softer spectrum. There is no evidence of flux variability during that period. A detailed publication on the analysis of these data is in preparation.

\section{Monitor known bright TeV blazars}

The improved sensitivity and energy threshold of MAGIC with respect to the former generation of IACTs allow a detailed study of the spectral features and flux variations of known TeV emitters. 
Mkn 421. Mkn 421 is the closest $\mathrm{TeV}$ blazar and the fi rst extragalactic VHE source ever detected. MAGIC has observed it for $25.6 \mathrm{~h}$, including $1.5 \mathrm{~h}$ of simultaneous observations with HESS. Integral flux level variations up to a factor four are observed between different observation nights, although no signifi cant intra-night variations have been recorded despite the high sensitivity of MAGIC for this kind of search. This flux variability in VHE gamma rays shows a positive correlation with X-ray data from the ASM instrument on-board the XRTE satellite. A flattening of the spectrum towards $100 \mathrm{GeV}$ of intrinsic origin has been observed, and suggest the presence of the IC peak predicted by the SSC model. Details of this analysis can be found in [10].

Mkn 501. This source is a close $\mathrm{TeV}$ blazar, and a very well studied object. MAGIC observed it during $55.0 \mathrm{~h}$, including $34 \mathrm{~h}$ in presence of moderate moonlight. This source was in a rather low state (30\%-50\% of the Crab Nebula flux above $200 \mathrm{GeV}$ ) during most of the observation period but showed two episodes of fast and intense flux variability, with doubling times of the order of 5 minutes. A spectral hardening following these flux variability has been observed, for the fi rst time in time scales of 10 minutes. A detailed publication on the analysis and results of these observations is in preparation.

1ES 1959+650. One of the main features displayed by this object is the observation of a flare in VHE gamma-rays without a counterpart in X rays [11], which cannot be explained by the SSC model for the acceleration mechanism in the jets. MAGIC observed this source during $6 \mathrm{~h}$ in its commissioning phase, resulting in a detection of gamma rays with a $8.2 \sigma$. Details at [12].

\section{Gamma Ray Bursts}

Its low energy threshold (50-100 GeV) and fast repositioning capability (less than 40s on average) make MAGIC a suitable instrument for the observation of the VHE prompt emission from GRBs. The minimum detectable flux after 1 (30) minutes of observation is 5.8 (1.1) Crab units above $80 \mathrm{GeV}$, and 1.8 (0.34) Crab units above $350 \mathrm{GeV}$. In Cycle 1, MAGIC followed up 11 GRBs, 2 of them while still in the prompt emission phase (see Table 1). No VHE emission was detected, and upper limits to the VHE flux were imposed. In Figure 2 we show the count rates of the BAT (15-350 keV) and MAGIC detectors together. No signifi cant excess over background is observed by MAGIC [13].

Table 1: GRBs observed by MAGIC during their prompt emission phase. $\Delta t_{a l}$ is the time between the burst onset and reception of the alert at the telescope, $\Delta t_{o b s}$ the total delay between the burst onset and the start of the MAGIC observation, $\mathrm{T}_{90}$ the total burst duration, $\Theta$ the mean zenith angle during the observation, $\mathrm{E}_{t h r}$ the energy threshold of the analyzed data and $z$ the redshift of the GRB. The last column shows the derived upper limits (in Crab units) to the GRB's fluence after different considered times (shown in the previous column) from the beginning of MAGIC's observation.

\begin{tabular}{cccccccccc}
\hline GRB ID & $\begin{array}{c}\text { onset } \\
{[\mathrm{UTC}]}\end{array}$ & $\Delta t_{a l}$ & $\Delta t_{o b s}$ & $\mathrm{~T}_{90}$ & $\Theta$ & $\begin{array}{c}\mathrm{E}_{t h r} \\
{[\mathrm{GeV}]}\end{array}$ & $z$ & $\begin{array}{c}\text { Obs Time } \\
{[\mathrm{s}]}\end{array}$ & $\begin{array}{c}\text { Fluence limit } \\
(\mathrm{Crab})\end{array}$ \\
\hline GRB050713a & $04: 29: 02$ & 13 & 40 & 70 & $49^{\circ}$ & 270 & $0.4-2.6$ & $90(1800)$ & $4.1(0.4)$ \\
GRB050904 & $01: 51: 44$ & 82 & 92 & 225 & $20^{\circ}$ & 95 & 6.3 & $133(1800)$ & $0.5(0.3)$ \\
\hline
\end{tabular}




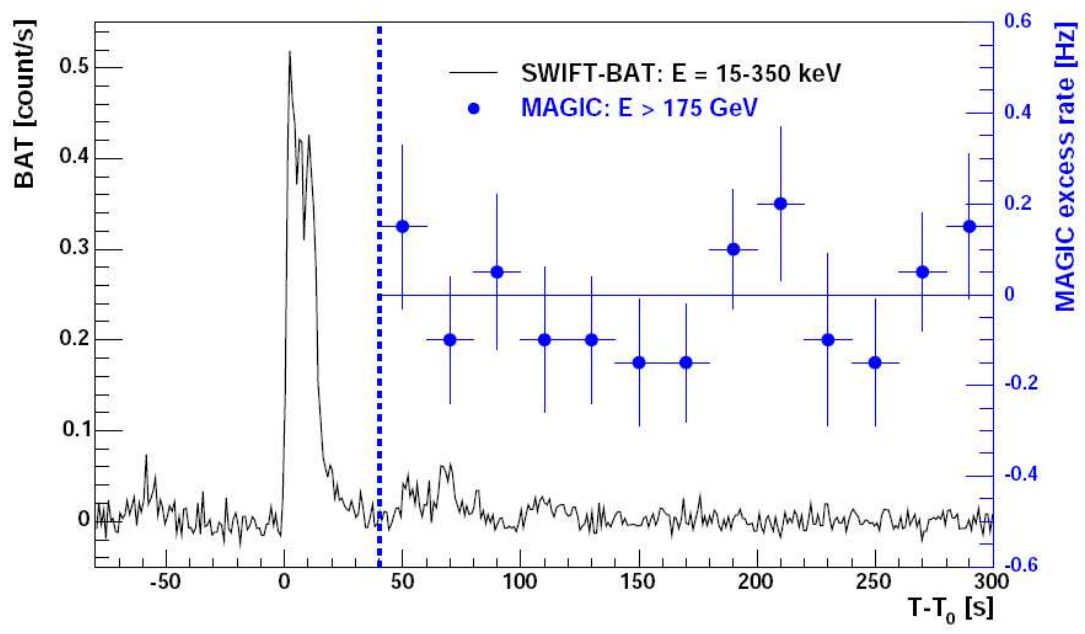

Figure 2: Emission time profile of GRB050713a by BAT and the event rate in $20 \mathrm{~s}$ bins from MAGIC. The energy range/threshold for the observation are indicated

\section{Conclusions}

MAGIC has concluded its fi rst operational campaign having detected seven extragalactic VHE gamma-ray sources, out of which three are new discoveries. The high sensitivity and low energy threshold allowed us to study the spectral features and the flux variability of these sources in great detail. For the fi rst time, an IACT is able to follow the prompt emission of GRBs. Two out of 11 GRB follow-up observations have been observed in the prompt emission phases. No signal could be seen so far and upper limits have been presented.

\section{References}

[1] P. Padovani and P. Giommi: ApJ 444567 (1995)

[2] L. Maraschi, G. Ghisellini and A. Celotti: ApJ 397 L5 (1992)

[3] D. Donato et al., A\&A 375739 (2001)

[4] L. Costamante and G. Ghisellini: A\&A 38456 (2002)

[5] K. Mannheim et al.: A\&A 31577 (1996)

[6] J. Albert et al: ApJ Letters 642 L119 (2006)

[7] F. Aharonian et al.: A\&A 448, L19 (2006)

[8] J. Albert et al: Submitted to ApJ Letters.

[9] J. Albert et al: ApJ Letters 648 L105 (2006)

[10] J. Albert et al: Submitted to ApJ.

[11] H. Krawczynski et al: ApJ 601151 (2004)

[12] J. Albert et al: ApJ 639761 (2006)

[13] J. Albert et al: ApJ 641 L9 (2006) 